\title{
OCORRENCIA DE OVOS DE Ancylostoma spp e Trichuris spp EM FEZES DE CÃES EM MEIA-PRAIA, ITAPEMA, SANTA CATARINA, BRASIL
}

\author{
Occurrence of Ancylostoma spp and Trichuris spp eggs in faeces of \\ dogs in Meia-Praia, Itapema, Santa Catarina, Brazil
}

\author{
Luiz Carlos Leite \\ Clarissa Ruhland Bandeira ${ }^{2}$ \\ Silvana Maris Cirio $^{3}$ \\ Ennio $\mathrm{Luz}^{4}$ \\ João Maria Ferraz Diniz ${ }^{5}$ \\ Selene CirioLeite 6 \\ DiegoLunelli ${ }^{7}$ \\ Stefany Weber ${ }^{8}$ \\ Claudia Regina Vieira Rocha Coelli ${ }^{9}$
}

\section{Resumo}

Entre abril e setembro de 2003, avaliou-se a ocorrência de ovos de Ancylostoma spp e Trichuris spp em fezes de cães, em Meia-Praia, Itapema, Santa Catarina, Brasil. Foram recolhidas 150 amostras de fezes, processadas no Laboratório de Parasitologia Veterinária do Departamento de Medicina Veterinária (PUCPR) sob os métodos laboratoriais de Willis (1) e Hoffmann et al. (2). Do total, 20 amostras (13,3\%) estavam contaminadas, sendo 17 (11,3\%) com ovos de Ancylostoma spp e 3 (2,0\%) com ovos de Trichuris spp. Estes resultados demonstram a necessidade do controle parasitário dos cães nesta região para diminuir o risco destas zoonoses.

Palavras-chave: Zoonoses; Ovos; Praia; Fezes; Cães.

\footnotetext{
${ }^{1}$ Médico Veterinário - Autônomo. e-mail: tudolica@pop.com.br

${ }^{2}$ Bióloga-Autônoma. e-mail: crbandeira@gmail.com

${ }^{3}$ Professora Doutora - Curso de Medicina Veterinária-CCAA - PUCPR. e-mail: silvana.cirio@pucpr.br

${ }^{4}$ Professor Titular - Depto. Patologia Básica UFPR. e-mail: sfzb@ufpr.br

${ }^{5}$ Professor Adjunto Aposentado - Depto. Medicina Veterinária UFPR. e-mail: dinis@ufpr.br

${ }^{6}$ Acadêmico - Curso de Medicina Veterinária - PUCPR. e-mail: lua_da_se@hotmail.com

${ }^{7}$ Acadêmico - Curso de Medicina Veterinária - PUCPR. e-mail: dilunelli@hotmail.com

${ }^{8}$ Acadêmico - Curso de Medicina Veterinária - PUCPR. e-mail: sweber@hotmail.com

${ }^{9}$ Acadêmico - Curso de Medicina Veterinária - PUCPR. e-mail: ccoelli@hotmail.com
} 


\section{Abstract}

From April to September of 2003, was evaluated the event of Ancylostoma spp and Trichuris spp eggs in dog faeces at Meia-Praia, Itapema, Santa Catarina, Brazil. Were collected 150 samples of faeces and processed in Veterinary Laboratory Parasitology of Veterinary Medicine Department (PUC-PR) under Willis (1) and Hoffmann et al. (2) methods. Were detected 20 positive samples $(13,3 \%)$, being 17 $(11,3 \%)$ for Ancylostoma spp. and 3 (2,0\%) for Trichuris spp. These results demonstrated the necessity of dogs parasitism control in this town for reduce the risk of these zoonoses.

Keywords: Zoonosis; Eggs; Beach; Faeces; Dogs.

\section{Introdução}

Os cães proporcionam inúmeros benefícios de natureza psicológica, fisiológica e social ao homem. De fundamental importância para o desenvolvimento emocional de crianças e bem-estar de seus proprietários, auxiliam indivíduos portadores de necessidades especiais e destacam-se como companhia para pessoas idosas. Conforme Vieira (3), o Brasil apresenta uma população estimada em vinte e cinco milhões de cães, a cidade de São Paulo registra quantidade superior a um milhão de cães e Curitiba alberga número superior a duzentos e oitenta mil cães.

De acordo com Scaini (4), Santarém et al. (5), Leite et al. (6), Dunn et al. (7), Guimarães et al. (8) Milano \& Oscherov (9), Castro et al. (10), Capuano \& Rocha (11) e Labruna et al. (12), paralelamente às vantagens advindas desta coexistência, outras situações configuram graves fatores de risco à saúde, pelo acentuado número de cães portadores de parasitas intestinais e com fácil acesso a locais de lazer, como acampamentos, áreas de recreação infantil, praias, praças, parques públicos e que freqüentemente defecam nestes locais, expondo freqüentadores a infecções, provocadas pelo contato de maneira direta ou indiretamente com fezes contaminadas por agentes parasitários. A eliminação continuada nas fezes, de milhares de ovos e cistos que permanecem viáveis por longos períodos no meio ambiente, poderá incorrer em infecções em outros cães, gatos e pessoas por meio da ingestão acidental de ovos larvados, cistos ou penetração ativa de larvas infectantes, denominadas larvas migrans cutâneas (LMC) que, após contato direto com a pele de seres humanos, geralmente desencadeiam dermatite acompanhada de prurido e erupções. Conhecida como "dermatite linear serpiginosa" ou "bicho geográfico", localiza-se freqüentemente nos membros inferiores, pés, nádegas, mãos e em menor intensidade na face e couro cabeludo. Conforme Kim et al. (13), freqüentemente é observado intenso prurido e o ato constante do indivíduo coçarse pode levar à instalação de infecções bacterianas secundárias nas partes atingidas. No Brasil, esta forma de doença tem como principais responsáveis as fases larvais de Ancylostoma caninum e Ancylostoma braziliense, helmintos descritos com relativa freqüência em cães e gatos $(5,8)$. Dois casos de larva migrans visceral (LMV) causada por Trichuris vulpis, normalmente encontrados parasitando cães, foram reportados em seres humanos por Sakano et al. (14). Mirdha et al. (15) comprovaram que o parasitismo por formas adultas de Trichuris vulpis em crianças e adultos pode desencadear úlceras duodenais, infecções intestinais acompanhadas de vômito, náusea, dor abdominal e diarréia crônica sanguinolenta ou com muco.

O presente trabalho teve por escopo confirmar a presença de ovos de Ancylostoma spp e Trichuris spp. em amostras de fezes de cães, coletadas em Meia-Praia, Itapema, estado de Santa Catarina, nos meses de abril a setembro de 2003.

\section{Materiais e métodos}

\section{Área do estudo}

Itapema está localizada a $27^{\circ} 05^{\prime} 25^{\prime \prime} \mathrm{S}$ e 48 36’41"W, no litoral do Estado de Santa Catarina, região Sul do Brasil (Figura 1). O município apresenta $59,022 \mathrm{~km}^{2}$ de área e é constituído por cinco praias (Praia da Ilhota, Costão de Itapema, Meia Praia, Central e Praia Grossa), perfazendo aproximadamente $7 \mathrm{~km}$ de litoral. Meia-Praia foi escolhida para o presente estudo por sua extensão $(3,5 \mathrm{~km})$, porcentagem considerável de cães observada à beira-mar e nas ruas e fluxo mensal de população flutuante em torno de 120 mil habitantes.

De acordo com dados do Instituto Brasileiro de Geografia e Estatística - IBGE (16) - sua população efetiva é de 28.750 habitantes. 

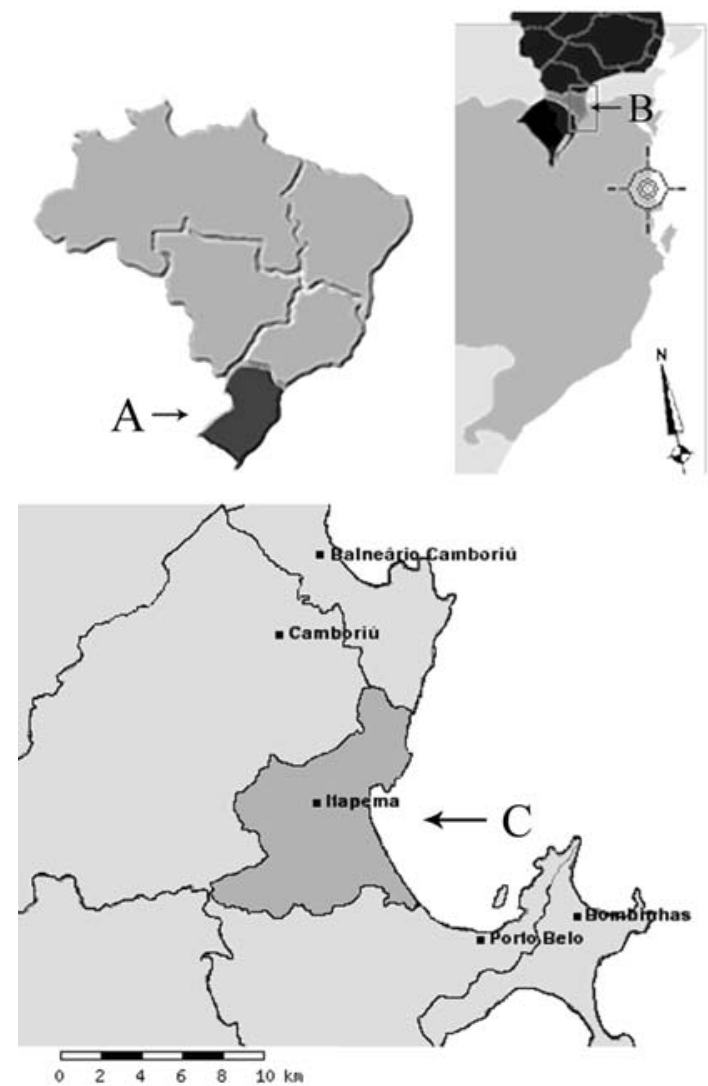

\section{Figura 1 - Localização da área de estudo \\ A - Região Sul do Brasil; B - Estado de Santa Catarina; C - Município de Itapema. Fonte: www.mapainterativo.ciasc.gov.br.}

\section{Metodologia}

A coleta das amostras foi realizada de abril a setembro de 2003, dividida em seis fases, diretamente da superfície da areia da praia e entrada de prédios e casas à beira-mar, locais previamente selecionados pela possibilidade de contaminação por matéria fecal e grande afluxo de pessoas e cães. De maneira aleatória, foram coletadas 150 amostras de fezes recentemente eliminadas por cães, as quais foram acondicionadas em frascos dotados de tampa, armazenadas em caixas de isopor contendo gelo reciclável, mantidas em refrigeração até a realização do exame e encaminhadas ao Laboratório de Parasitologia Animal do Curso de Medicina Veterinária da PUCPR, em São José dos Pinhais - PR, para análise em um período máximo de 24 horas após a coleta pelos métodos de Willis (1) de flutuação em solução salina e de Hoffmann et al. (2). Todas as amostras foram observadas ao microscópio óptico, inicialmente em objetiva de 10x e com posterior confirmação em objetiva de 40x. Os ovos encontrados foram classificados de acordo com suas características morfológicas, visando a identificação do gênero dos parasitas. Não foi possível no presente trabalho fazer uma diferenciação dos ovos entre as espécies Ancylostoma braziliense e Ancylostoma caninum.

\section{Resultados e discussão}

Com respeito aos resultados da análise de 150 amostras de fezes de cães para a detecção de ovos de helmintos, foram identificadas amostras contaminadas por ovos de Ancylostoma spp e Trichuris spp., sendo observadas $17(11,3 \%)$ amostras positivas para Ancylostoma spp e $3(2,0 \%)$ amostras positivas para Trichuris sp (Tabela 1). 
Tabela 1 - Ocorrência de ovos de Ancylostoma spp E Trichurisspp em amostras de fezes de cães coletada em meia-praia, Santa Catarina, Brasil

\begin{tabular}{ccccccc}
\hline \multicolumn{2}{c}{ Dados da Coleta } & \multicolumn{2}{c}{ Ancylostoma spp } & \multicolumn{2}{c}{ Trichuris spp } \\
\hline $\begin{array}{c}\mathbf{N}^{\circ} \text { da } \\
\text { coleta }\end{array}$ & Data & $\begin{array}{c}\mathbf{N}^{\circ} \text { de } \\
\text { amostra }\end{array}$ & Positivas & $\%$ & Positivas & $\%$ \\
& & $\mathbf{s}$ & & & & \\
\hline $\mathbf{1}$ & $04 / 04 / 03$ & 11 & 4 & 36,4 & 0 & 0 \\
$\mathbf{2}$ & $20 / 04 / 03$ & 24 & 4 & 16,7 & 0 & 0 \\
$\mathbf{3}$ & $18 / 05 / 03$ & 19 & 3 & 15,8 & 0 & 0 \\
$\mathbf{4}$ & $31 / 05 / 03$ & 36 & 3 & 8,3 & 1 & 2,7 \\
$\mathbf{5}$ & $17 / 06 / 03$ & 30 & 1 & 3,3 & 1 & 3,3 \\
$\mathbf{6}$ & $05 / 09 / 03$ & 30 & 2 & 6,7 & 1 & 3,3 \\
\multicolumn{2}{c}{ TOTAL } & $\mathbf{1 5 0}$ & $\mathbf{1 7}$ & $\mathbf{1 1 , 3} \%$ & $\mathbf{3}$ & $\mathbf{2 , 0} \%$ \\
\hline
\end{tabular}

Os resultados demonstraram maior prevalência de ovos de Ancylostoma spp em relação a ovos de Trichuris spp. Apesar de a amostragem ser determinada de maneira aleatória, o presente trabalho comprovou, a exemplo do observado por vários autores, a presença de Ancylostoma como o gênero de helminto diagnosticado em maior porcentagem em fezes de cães no Brasil $(6,11,12,22)$. Duas amostras analisadas na primeira coleta e quatro amostras da segunda coleta continham ovos larvados de Ancylostoma spp, comprovando a possibilidade de infecção por larva migrans cutânea (LMC).

Índices superiores de contaminação em relação ao trabalho em epígrafe foram obtidos por diversos autores, em estudos realizados no litoral do estado de São Paulo. Nunez et al. (17), em Peruíbe, obtiveram índice de positividade de $50(74,63 \%)$ amostras de Ancylostoma caninum e de $20(13,43 \%)$ para Trichuris vulpis em 150 amostras de fezes analisadas. Levantamento epidemiológico realizado por Souza et al. (18) em Santos, encontrou 81 (96,4\%) amostras positivas para ovos de Ancylostoma sp e $3(3,5 \%)$ contaminadas por ovos de Trichuris sp em 150 amostras fecais avaliadas. Pereira et al. (19) avaliaram 150 amostras de fezes de cães em São Vicente, encontrando percentual de positividade em $74(49,2 \%)$ delas para Ancylostoma sp e em 20 $(13,4 \%)$ amostras contaminadas com ovos de
Trichuris sp. Genaro et al. (20) em Praia Grande demonstraram presença de ovos de Ancylostoma caninum em $26(81,25 \%)$ amostras de fezes de um total de 100 estudadas. Ainda em Praia Grande, a análise de 257 amostras de fezes confirmaram a contaminação em 118 (45,9\%) por ovos de Ancylostoma sp. (10). Genaro et al. (21), em Monganguá, avaliaram 150 amostras fecais de cães e obtiveram índice de contaminação por Ancylostoma sp. em $62(69,6 \%)$ delas. No estado do Rio Grande do Sul, em balneário Cassino, também foram obtidos índices superiores de positividade, encontrando-se ovos de Ancylostoma spp. em 103 (43,5\%) e de Trichuris spp em 23 (9,7\%) de 237 amostras de fezes de cães analisadas (4). Igualmente, no Rio Grande do Sul, na praia de Ipanema, Matesco et al. (22) obtiveram de 121 amostras de fezes de cães analisadas, 39 positivas para ovos dos gêneros Ancylostoma spp (29,8\%) e Trichuris spp (3,3\%). Na cidade de Mar del Plata, Argentina, Rodriguez et al. (23) examinaram 205 amostras de fezes de cães, sendo que $139(67,8 \%)$ apresentaram ovos de Ancylostoma caninum e $106(52,1 \%)$ de Trichuris vulpis. Em Taiwan, Ho et al. (24) obtiveram 30 (8,0\%) amostras positivas para Ancylostoma caninum e $12(3,2 \%)$ contaminadas por Trichuris vulpis de um total de 376 amostras de fezes de cães examinadas. Em Nápoles, região sul da Itália, 
Rinaldi et al. (25) examinaram 415 amostras de fezes e encontraram índices de positividade para Ancylostoma caninum em $10(2,4 \%)$ e de Trichuris vulpis em $42(10,1 \%)$.

\section{Conclusão}

A partir dos resultados obtidos, conclui-se que a sanidade das praias é de suma importância para a saúde pública, visto o grande potencial de risco gerado na transmissão de zoonoses nestes locais causadas por helmintos parasitas de cães e gatos, especialmente a larva migrans cutânea (LMC). Os dados registrados reforçam a necessidade de ações preventivas e medidas de controle sanitário dessas áreas, com o propósito de evitar a presença de animais, sejam eles domésticos ou errantes e conseqüentemente o acúmulo de dejetos nas praias. Igualmente, os cães errantes assumem grande importância na manutenção e disseminação de zoonoses, uma vez que geralmente são excluídos de quaisquer estatísticas e programas de educação sanitária e controle parasitário (12). Torna-se necessário também a implantação de campanhas de conscientização da população local e dos turistas por meio dos profissionais de Medicina Veterinária, ressaltando a importância de tratamento antihelmíntico regular em cães e gatos e reforçando o compromisso social de posse responsável dos proprietários para restringir ao máximo a contaminação ambiental e os riscos de infecção humana e canina.

\section{Agradecimentos}

Às pessoas que auxiliaram neste trabalho: Marcos Vidor Bandeira, Iara Ruhland Bandeira, Flávio Soares, Melissa Ruhland Bandeira e Raphael Eduardo Fernandes Santos.

\section{Referências}

1. Willis HH. A simple levitation method for the detection of hookworm ova. Med J Australia, North. 1921; 8:375-376.

2. Hoffmann WA, Pons JA, Janer JL. Sedimentationconcentration method in Schistomiasis mansoni. J Pub Health Trop Med. 1934; 9:283-298.

3. Vieira HRA. Curitiba e os cães: Uma contribuição para o debate. Rev do CRMV PR. 2003; 8:17-21.
4. Scaini CJ, Toledo RN, Lovatel R, Dionello MA, Gatti FA, Susin L, et al. Contaminação ambiental por ovos e larvas de helmintos em fezes de cães na área central do Balneário Cassino, Rio Grande do Sul. Rev Soc Bras Med Trop. 2003; 36(5):617-619.

5. Santarém VA, Giuffrida R, Zanin GA. Larva migrans cutânea: ocorrência de casos humanos e identificação de larvas de Ancylostoma spp. em parque público do município de Taciba, São Paulo. Rev Soc Bras Med Trop. 2004; 37(2):179-181.

6. Leite LC, Marinoni LP, Cirio SM, Diniz JMF, Silva MAN, Luz E, et al. Endoparasitas em cães (Canis familiaris) na cidade de Curitiba-Paraná - Brasil. Arch vet Sci. 2004; 9:95-99.

7. Dunn JJ, Columbus ST, Aldeen WE, Davis M, Carrol KC. Trichuris vulpis recovered from a patient with chronic diarrhea and five dogs. J Clin Microb. 2002; 40(7):2703-2704.

8. Guimarães AM, Alves EGL, Rezende GF, Rodrigues MC. Ovos de Toxocara sp. e larvas de Ancylostoma sp. em praça pública de Lavras, MG. Rev Saúde Publ. 2005; 39(2):293-295.

9. Milano AMF, Oscherov EB. Contamiación de aceras com enteroparasitos cani-nos em Corrientes, Argentina. Parasitol Latinoam. 2005; 60:82-85.

10. Castro JM, Santos SV, Monteiro NA. Contaminação de canteiros da orla marítima do Município de Praia Grande, São Paulo, por ovos de Ancylostoma e Toxocara em fezes de cães. Rev da Soc Bras de Med Trop. 2005; 38(2):199-201.

11. Capuano DM, Rocha GM. Ocorrência de parasitas com potencial zoonótico em fezes de cães coletadas em áreas públicas do município de Ribeirão Preto, Brasil. Rev Bras Epidemiol. 2006; 9(1):81-86.

12. Labruna MB, Pena HFJ, Souza SLP, PinterA, Silva JCR, RagozoAMAetal. Prevalência de endoparasitas em cães da área urbana do município de Monte Negro, Rondônia. Arq Inst Biol. 2006; 73(2):183-193.

13. Kim TH, Lee BS, Sohn WM. Three cases of cutaneous larva migrans. Korean J. Parasit. 2006; 44(2):145-149.

14. Sakano T,Hamamoto K, KobayashiY, SakataY, Tsuji M, Usui T. Visceral larva migrans caused by Trichuris vulpis. Arch Dis Childhood. 1980; 55:631-633.

15. Mirdha BR, Singh IG, Samantray JC, Mishra B. Trichuris vulpis infection in slum children. Indian J Gastroent. 1998; 17(4):154-154. 
16. Instituto Brasileiro de Geografia e Estatítica-IBGE. Resolução 05, 10 de outubro de 2002. Área Territorial -UF - Santa Catarina, Brasil. [atualizado 2006; citado 2006 jun 15]. Disponível em: URL: http://www.ibge.gov.br

17. Nunez MV, Portaleoni F, Certo CMG, Rodrigues AC. Levantamento parasitológico em cães (Canis familiaris) domiciliados e semi domiciliados no município de Peruíbe, Baixada Santista. In: Reunião Anual do Instituto Biológico, XVII. São Paulo. Resumos... Arq Inst Biol. 2004; 71(supl.):70-70.

18. Souza MF, Evangelista TFS, Santos TS, Barros MEP, Serôdio JJ, Conceição TM et al. Presença de ovos de Ancylostoma sp. nas fezes de cães (Canis familiaris) domiciliados no bairro Aparecida, município de Santos, São Paulo, Brasil. In: Congresso de Iniciação Científica em Ciências agrárias, Biológicas e Ambientais, III. São Paulo. Resumos... Arq Inst Biol. 2005; 72(supl.1): 39-39.

19. Pereira CEM, Fontoura MFG, Alvez de Souza PA, Rodrigues AC. Ocorrência de ovos e oocistos de parasitas gastrointestinais nas fezes de cães (Canis familiaris) recolhidas em vias públicas no bairro Jockey Club, município de São Vicente, São Paulo, Brasil. In: $3^{\circ}$ Congresso de Iniciação Científica em Ciências agrárias, Biológicas eAmbientais. São Paulo. Arq Inst Biol. 2005; 72(supl.1):39-39.

20. Genaro T, Secco de Carvalho VCM, Silva NA, Rodrigues AC. Endoparasitos intestinais em cães (Canis familiaris) domiciliados no município de Praia Grande, Baixada Santista. In: $7^{\circ}$ Reunião Anual do Instituto Biológico. São Paulo. Arq Inst Biol. 2004; 71(supl.):70-70.
21. Genaro T, Secco de Carvalho VCM, Statch D, Mattos AM, Rodrigues AC, Capatto K. Ancylostoma sp. em cães (Canis familiaris) do Centro de Controle de Zoonoses de Mongaguá, Baixada Santista, São Paulo, Brasil. In: $3^{\circ}$ Congresso de Iniciação Científica em Ciências agrárias, Biológicas e Ambientais. São Paulo. Arq Inst Biol. 2005; 72(supl.1):38-38.

22. Matesco VC, Mentz MB, Rott MB, Silveira CO. Contaminação sazonal por ovos de helmintos na praia de Ipanema, em Porto alegre, Rio Grande do Sul, Brasil. Rev Patol Trop. 2006; 35(2):135-141.

23. Rodriguez F, Denegri G, Sardella N, Hollmann P. Relevamiento coproparasitológico de caninos ingresados al centro Municipal de Zoonosis de Mar del Plata, Argentina. Rev Vet. 2005; 16(1):9-12.

24. Ho SH, Watanabe Y, Lee YC, Shih TH, Tu WJ, Ooi HK. Survey of Gastrointestinal Parasitic Infections in Quarantined Dogs in Taiwan. J Vet Med Sci. 2006; 68(1):69-70.

25. Rinaldi L, Biggeri A, Carbone S, Musella V, Catelan D, Veneziano V, Cringoli G. Canine faecal contmination and parasitic risk in the city of Naples (southern Italy). BioMedCentral Vet Res. [serial on the Internet]. 2006 [cited 2006 jun 10]; 2:29. available from: URL:

http://www.biome-central.com/1746-6148/2/29

Recebido em/Received in: July 28, 2006 Aceito em/Accepted in: August 31, 2006 